\title{
Two-Hour Lactate Clearance Predicts Negative Outcome in Patients with Cardiorespiratory Insufficiency
}

\author{
Sean Scott, ${ }^{1}$ Vittorio Antonaglia, ${ }^{2}$ Giovanna Guiotto, ${ }^{3}$ Fiorella Paladino, ${ }^{3}$ \\ and Fernando Schiraldi ${ }^{3}$
}

${ }^{1}$ Emergency Department, Royal North Shore Hospital, Sydney, Australia
${ }^{2}$ Department of Anaesthesia and Intensive Care, University of Trieste, Trieste 34123, Italy
${ }^{3}$ Department of Emergency Medicine and Critical Care, San Paolo Hospital, Naples, Italy

Correspondence should be addressed to Fernando Schiraldi, schirald@libero.it

Received 23 September 2009; Accepted 23 May 2010

Academic Editor: Robert Boots

Copyright (c) 2010 Sean Scott et al. This is an open access article distributed under the Creative Commons Attribution License, which permits unrestricted use, distribution, and reproduction in any medium, provided the original work is properly cited.

\begin{abstract}
Objective. To evaluate 2-hour lactate clearance as a prognostic marker in acute cardiorespiratory insufficiency. Design. Prospective observational study. Setting. Emergency Department (ED) and 16-bed medical High Dependency Unit (HDU). Methods and Main Results. 95 consecutive admissions from the ED for acute cardiorespiratory insufficiency were prospectively enrolled. Arterial lactate concentration was assessed at ED arrival and 1, 2, 6, and 24 hours later. The predictive value of 2-hour lactate clearance was evaluated for negative outcomes defined as hospital mortality or need for endotracheal intubation versus positive outcomes defined as discharge or transfer to a general medical ward. Logistic regression and ROC curves found 2-hour lactate clearance $>15 \%$ was a strong predictor of negative outcome $(P<.0001)$ with a sensitivity of $86 \%(95 \% \mathrm{CI}=67 \%-95 \%)$ and a specificity of $91 \%(95 \% \mathrm{CI}=82 \%-96 \%)$, Positive predictive value was $80 \%(95 \% \mathrm{CI}=61 \%-92 \%)$, and negative predictive value was $92 \%$ $(95 \% \mathrm{CI}=84 \%-98 \%)$. Conclusions. Systematic monitoring of lactate clearance at 2 hours can be used in to identify patients at high risk of negative outcome and perhaps to tailor more aggressive therapy. Equally important is that a 2-hour lactate clearance $>15 \%$ is highly predictive of positive outcome and may reassure clinicians that the therapeutic approach is appropriate.
\end{abstract}

\section{Introduction}

Acute cardiorespiratory failure from any cause creates an imbalance between energy supply, demand, and consumption. A high lactate concentration associated with a low blood $\mathrm{pH}$ is useful to depict the gravity of such a mismatch [1]. Lactate overproduction can be promptly removed from blood if renal and hepatic perfusion and function are near normal $[2,3]$. A huge bulk of research has been focused on lactate production/removal in critically ill patients. Some evidence suggests that persistent elevation in lactate is associated with high mortality rate [4-6]. It is generally agreed that the longer lactate levels remain high, the worse the prognosis $[7,8]$. However, a question remains regarding the timing needed to guide the therapy based on evaluation of lactate clearance [9].

Following the concept of "Early-Goal-Directed-Therapy" (EGDT) [10], one should be satisfied if the therapeutic approach determines a lactate clearance greater than $20 \%$ within 6 hours of treatment (LACT-6h-clearance). Indeed, this metabolic goal is acceptable when the lactate overproduction reflects a hypermetabolic state, as in septic patients. However, LACT-6h-clearance is less useful in assessing the response to treatment when an increase in lactate occurs rapidly, as in patients with acute cardiorespiratory failure.

We hypothesized that, in these patients, an earlier lactate clearance should be useful to assess the metabolic response to therapy and to identify patients able to reverse the metabolic derangement. A secondary hypothesis was that failure to rapidly resolve the oxygen debt, indicated by poor lactate clearance, would be correlated with negative outcome. For this purpose a prospective series of patients consecutively admitted to the Emergency Department with acute cardiorespiratory failure underwent a standardized therapeutic approach and the metabolic response was assessed by lactate 
clearance after 2 hours of treatment (LACT-2h-clearance). The first aim of the study was to evaluate the utility of short-term lactate clearance in acute cardiorespiratory failure patients and the second was to assess its value in predicting patient outcome.

\section{Materials and Methods}

2.1. Inclusion Criteria. Acute decompensated cardiac failure and/or "acute-on-chronic" respiratory failure. Acute cardiac failure was defined as a condition with decreased tissue perfusion other than simple hypovolemia in which volume and/or drugs that improve cardiac performance and/or restore abnormal vascular tone could be prescribed. Respiratory failure was defined as oxygen saturation of less than $90 \%$ despite supplementary oxygen with or without hypercapnia.

These entities were defined clinically in the emergency department with at least 4 out of 6 of the following parameters:

Respiratory rate $>25$

Heart rate $>130$

Systolic blood pressure $<90$

$\mathrm{SpO}_{2}<90 \%$ at $\mathrm{FiO}_{2}$ of 0.30

Bibasal crepitations on auscultation

Arterial blood lactate $>2 \mathrm{mmol} / \mathrm{L}$.

\subsection{Exclusion Criteria}

Pneumonia defined as

lung infiltrate on the chest radiograph associated with at lest two of the following conditions: fever, leucocytosis, purulent sputum in which a Gram stain showed one or more types of bacteria.

Sepsis syndrome defined as

positive blood cultures and/or fever of $>38^{\circ} \mathrm{C}$ together with positive cultures from suspected sources.

Renal dialysis

Glasgow coma scale $<8$

Criteria for immediate intubation for cardiopulmonary resuscitation

Inotropic support with catecolamines at baseline

Failure of two or more additional organs defined as

Bilirubin $>2 \mathrm{mg} / \mathrm{dL}$, creatinine $>2 \mathrm{mg} / \mathrm{dL}$ or creatinine clearance $<50 \mathrm{~mL} / \mathrm{min}$, platelets $<100.000 / \mathrm{mmc}, \mathrm{Hb}<7.5 \mathrm{~g} / \mathrm{dL}$.

Electrocardiogram instability with evidence of lifethreatening ventricular arrhythmia.
2.3. Patients and Study Design. Ninety five consecutive patients with acute cardiorespiratory insufficiency, admitted from an emergency department to a 16-bed medical ICU/HDU during 2008, were prospectively enrolled in the study. Anthropometric and functional characteristics of the patients are listed in Table 1.

The preliminary diagnosis was made according to clinical history, physical examination, arterial blood gas, and chest radiography. Subsequent investigations and monitoring took place in the Medical Intensive Care or High Dependency Unit (ICU/HDU) depending on acuity. The following data were collected for all patients. Blood pressure, heart and respiratory rate, Glasgow Coma Scale [11] and APACHE II score [12], arterial blood gases and lactate, full blood count, biochemistry panel, ECG monitoring, and ecocardiographic evaluation.

\section{Interventions}

Gas exchange and lactate blood concentration were assessed at ED arrival and 1, 2, 6, and 24 hours later. Two-hour lactate clearance was calculated as

$$
\frac{\text { (Lactate start - lactate } 2 \text { hour) }}{\text { lactate start (\%) }} .
$$

Blood gas-analysis and arterial lactate were performed by intermittent blood sampling and co-oximetry (Blood Gas Analyzer, Bayer Health Care Rapid Lab 1265). Inspired fractional $\mathrm{O}_{2}$ concentration $\left(\mathrm{FiO}_{2}\right)$ was recorded.

All patients were treated according to standard practice in our ICU. Protocols for hemodynamic treatment including inotropic support with dobutamine or phosphodiesterase inhibitors, noninvasive positive pressure ventilation (NPPV), light sedation, management of blood glucose, water, and electrolyte derangements were used. No patients in our study were treated with lactate containing solutions, which might have falsely elevated lactate levels.

The criteria for patients who underwent NPPV were tachypnea with a respiratory rate greater than $25 \mathrm{bpm}$, $\mathrm{pH}$ 7.10-7.35, and $\mathrm{PaCO}_{2}>50 \mathrm{~mm} \mathrm{Hg}$. Continuous or bilevel positive pressure support were chosen at the discretion of the treating physician and adapted to response of the patient. The criteria to determine when ventilator assistance was discontinued were normal mental status, hemodynamic stability, respiratory rate below $25 \mathrm{bpm}$, absence of activation of accessory respiratory muscles and paradoxical abdominal motion, arterial $\mathrm{pH}>7.33, \mathrm{PaCO}_{2}<70 \mathrm{mmHg}, \mathrm{PaO}_{2}>$ $55 \mathrm{mmHg}$ with $\mathrm{FIO}_{2}>0.35$ without ventilatory support. The patients were discharged from the ICU/HDU when NPPV was switched to oxygen therapy with $\mathrm{FIO}_{2}<0.35$ for $24 \mathrm{~h}$ at least and the patients met the following criteria: cardiorespiratory stability, respiratory rate below $25 \mathrm{bpm}$, $\mathrm{pH}>7.35, \mathrm{PaO}_{2}>55 \mathrm{mmHg}, \mathrm{PaCO}_{2}<70 \mathrm{mmHg}$, absence of fever, leucocytosis, purulent sputum, and metabolic complications. An independent clinical team not involved in the research protocol decided about the discharge.

Primary outcome measures were hospital mortality or endotracheal intubation (negative outcome) and discharge or transfer to a general medical ward (positive outcome). 


\section{Statistical Analysis}

The data are expressed as mean and standard deviation for continuous variables, medians, and interquartile range (1st-3rd) for ordinal variables and numbers and frequencies for nominal variables. Normality of data distribution was appraised with Kolmogorov-Smirnov test. Homoscedicity was tested with Levene median test with Lilliefors correction. Based on pilot data and previous works on the prognostic value of lactate in the critically ill $[4,10,13]$, the study was powered for an expected sensitivity of $90 \%$ with a minimal acceptable confidence limit of 75\% [14, 15].

Factors likely to affect outcome were recorded at the outset. These included age $>70$ years, presence of severe underlying diseases (cancer, pre-existing hepatic, or renal insufficiency) presence of shock, acute renal failure, and baseline laboratory parameters. Factors correlated with negative outcome at $P<.25$ (chi-square or Fisher's exact test, as appropriate) were included in a logistic regression model with the lactate clearance variables. A backward procedure was then used to discard the nonsignificant variables from the analysis. Sensitivity, specificity, and predictive values were calculated according to standard formulae. Receiver operating characteristics curves (ROC) were constructed to evaluate the reliability of 2-hour lactate clearance as prognostic factor. $P<.05$ was considered significant. All statistical analyses were performed with SPSS 15.0 for windows.

\section{Results}

A total of 95 patients were included in the study. All patients had a history of significant underlying pathology, sixtythree had ischemic heart disease $(68 \%)$, thirty-nine patients had COPD (42\%), thirty-five had type 2 diabetes (42\%), and twelve had chronic renal failure (8.7\%). At enrolment 56 patients were in acute pulmonary oedema (68\%), 25 patients had acute respiratory failure for severe exacerbation of COPD (21\%), 7 were in cardiogenic shock $(6 \%)$, and 5 patients presented with acute myocardial infarction. 34 patients required noninvasive positive pressure ventilation (NPPV) (43\%). The overall negative outcome rate was 30\% (28/95) 12\% hospital mortality (11/95). 25 patients required endotracheal intubation and of those $10(40 \%)$ died during their hospital stay. Lactate at baseline was not different between groups but 2-hour lactate and 2-hour lactate clearance were significantly worse in patients with negative outcomes (Table 1).

The odds ratio for both elevated 2 hour lactate $(7.73, P=$ .002) and impaired LACT-2h-clearance $(16.11, P<.0001)$ are highly significant for negative outcome but LACT-2hclearance appears superior. The odds ratios of selected risk factors are displayed in Table 2.

Figure 1 illustrates ROC curves for LACT-2h-clearance. It demonstrates the reliability of LACT-2h-clearance as a predictor of negative outcome, indicating that the best compromise between sensitivity and specificity was obtained for a lactate clearance of $15 \%$. The global reliability of this test to predict mortality is quite good, as confirmed by the

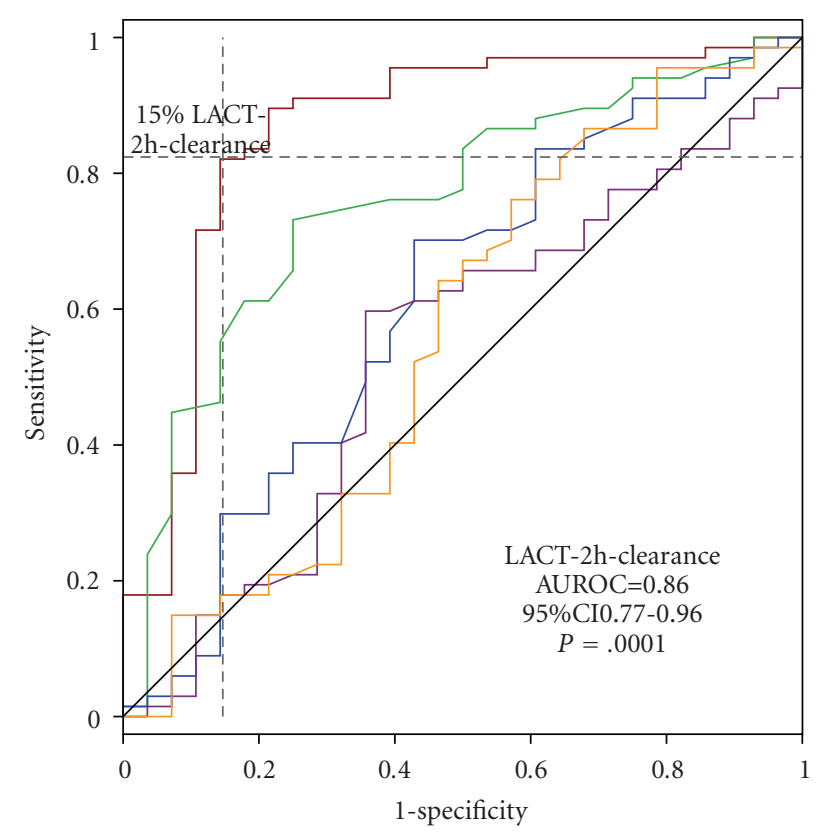

Source of the curve

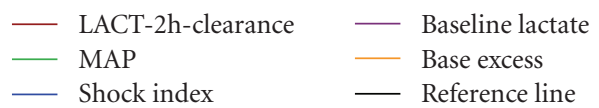

Figure 1: ROC curve for LACT-2h-clearance.

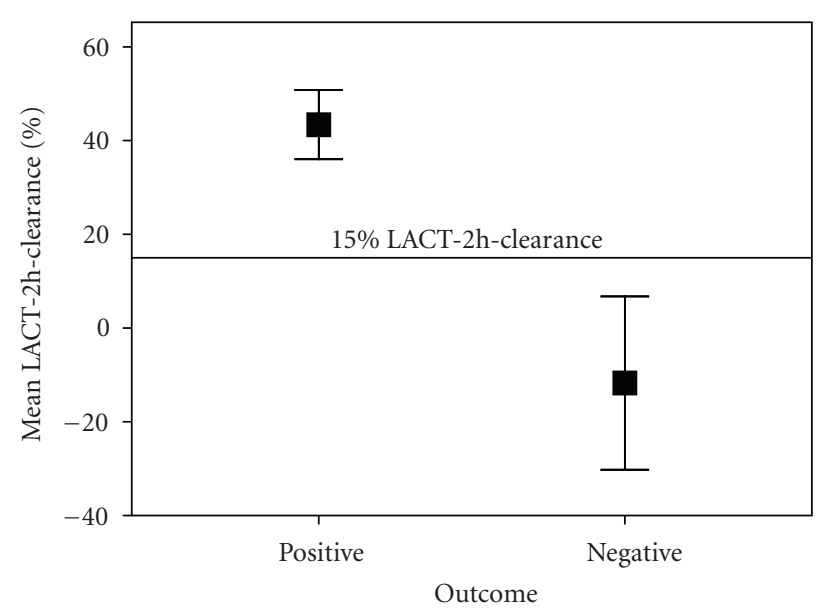

Error bars: 95\% CI

FIGURE 2: Mean LACT-2h-clearance for positive and negative outcome.

value of the area under the ROC (AUROC) curve of 0.86 ( $P \leq$ $.0001 ; 95 \%$ CI $0.77-0.96)$ which is comparable to the values previously reported for other risk factors of mortality in similar studies [13]. Figure 2 displays a graphical comparison of mean LACT-2h-clearance between patients with positive and negative outcomes.

When $<15 \%$ is used as a cut off, LACT-2h-clearance accurately predicted negative outcome with a sensitivity 
TABLE 1: Patient characteristics.

\begin{tabular}{|c|c|c|c|c|}
\hline Variables & Patients $(n=95)$ & Positive outcome $(n=67)$ & Negative outcome $(n=28)$ & $P$ \\
\hline Age $($ mean $\pm \mathrm{SD})$ & $76.1 \pm 9.4$ & $75.5 \pm 9.2$ & $77.8 \pm 9.8$ & NS \\
\hline $\mathrm{M} / \mathrm{F}$ & $43 / 52$ & $28 / 39$ & $5 / 8$ & NS \\
\hline Apache II score & $19.4 \pm 4.6$ & $19.3 \pm 4.2$ & $19.8 \pm 5.3$ & NS \\
\hline \multicolumn{5}{|c|}{ Vital parameters } \\
\hline Heart rate bpm & $114 \pm 30.2$ & $117.3 \pm 27.8$ & $106.0 \pm 34.7$ & NS \\
\hline Respiratory rate & $34.4 \pm 6.8$ & $35.4 \pm 6.3$ & $32.3 \pm 7.6$ & NS \\
\hline MAP mmHg & $113.5 \pm 32.8$ & $115.6 \pm 29.1$ & $94.0 \pm 29.3$ & $P<.05^{*}$ \\
\hline \multicolumn{5}{|c|}{ Laboratory values } \\
\hline $\mathrm{WBC}, \mathrm{mm}^{3}$ & $13.3 \pm 5.4$ & $13.3 \pm 5.3$ & $13.0 \pm 5.8$ & NS \\
\hline HTC, \% & $37.8 \pm 7.5$ & $38.5 \pm 7.2$ & $34.2 \pm 8.2$ & NS \\
\hline Creatinine, $\mathrm{mg} / \mathrm{dL}$ & $1.37 \pm 0.6$ & $1.36 \pm 0.75$ & $1.43 \pm 0.3$ & NS \\
\hline $\mathrm{pH}$ & $7.25 \pm 0.14$ & $7.24 \pm 0.08$ & $7.26 \pm 0.16$ & NS \\
\hline $\mathrm{pO}_{2}, \mathrm{mmHg}$ & $63.4 \pm 22.5$ & $67.0 \pm 33.1$ & $61.9 \pm 16.3$ & $P<.05^{*}$ \\
\hline $\mathrm{pCO}_{2}, \mathrm{mmHg}$ & $51.4 \pm 20.6$ & $51.1 \pm 17.6$ & $52.8 \pm 26.6$ & NS \\
\hline $\mathrm{HCO}_{3}, \mathrm{mEq} / \mathrm{L}$ & $21.5 \pm 5.8$ & $21.2 \pm 5.1$ & $22.5 \pm 7.1$ & NS \\
\hline Lactate start, $\mathrm{mmol} / \mathrm{L}$ & $4.7 \pm 2.6$ & $4.7 \pm 2.4$ & $4.7 \pm 2.9$ & NS \\
\hline Lactate $2 \mathrm{~h}, \mathrm{mmol} / \mathrm{L}$ & $3.0 \pm 2.0$ & $2.2 \pm 0.9$ & $4.7 \pm 2.8$ & $P<.05^{*}$ \\
\hline Lactate clearance at 2 hours $(\%)$ & $27 \pm 43$ & $43 \pm 30$ & $-11 \pm 47$ & $P<.05^{*}$ \\
\hline
\end{tabular}

${ }^{*} P<.05$, Student $T$-test; NS, Not Significant.

MAP: mean arterial pressure, WBC: white blood count, HTC: hematocrit.

TABLE 2: Relationship between risk factors and negative outcome.

\begin{tabular}{|c|c|c|c|c|}
\hline Variables & Positive outcome $(n=67)$ & Negative outcome $(n=28)$ & Odds Ratio $(95 \% \mathrm{CI})$ & $P$ \\
\hline Age $>75$ & $41(61 \%)$ & $20(71 \%)$ & $1.59(0.79-3.17)$ & .34 \\
\hline Male/Female & $28 / 39(42 / 58 \%)$ & $15 / 13(54 / 46 \%)$ & N/A & .57 \\
\hline IHD & $47(29 \%)$ & $16(31 \%)$ & $0.57(0.27-1.16)$ & .23 \\
\hline Cardiogenic shock & $3(5 \%)$ & $4(14 \%)$ & $3.55(0.74-16.93)$ & .07 \\
\hline Myocardial Infarction & $4(6 \%)$ & $1(4 \%)$ & $0.59(0.06-5.45)$ & .67 \\
\hline COPD & $29(44 \%)$ & $10(38 \%)$ & $0.73(0.31-1.69)$ & .49 \\
\hline Renal Failure & $10(32 \%)$ & $2(46 \%)$ & $0.44(0.09-2.13)$ & .31 \\
\hline $\mathrm{p} 0_{2}<60$ at 2 hours & $7(17 \%)$ & $3(15 \%)$ & $1.03(0.25-4.26)$ & .96 \\
\hline Hemaglobin $<10 \mathrm{~g} / \mathrm{dL}$ & $5(7 \%)$ & $8(29 \%)$ & $4.10(1.21-13.86)$ & .02 \\
\hline $\mathrm{p} 0_{2}<60 \mathrm{mmHg}$ at presentation & $34(38 \%)$ & $17(84.6)$ & $1.50(0.72-3.11)$ & .005 \\
\hline MAP $<90$ at presentation & $9(13 \%)$ & $13(46 \%)$ & $5.58(2.01-15.52)$ & .001 \\
\hline Serum lactate $>2.5 \mathrm{mmol} / \mathrm{L}$ at 2 hours & $23(37 \%)$ & $25(82 \%)$ & $7.73(2.60-22.90)$ & .0002 \\
\hline Lactate clearance $<20 \%$ at 2 hours & $9(21 \%)$ & $20(69.2)$ & $16.11(6.53-39.70)$ & $<.0001$ \\
\hline
\end{tabular}

IHD-ischemic heart disease; COPD-chronic obstructive pulmonary disease; CI-confidence interval.

of $86 \%(95 \% \mathrm{CI}=67 \%-95 \%)$ and a specificity of $91 \%$ $(95 \% \mathrm{CI}=82 \%-96 \%)$. Positive predictive value was $80 \%$ $(95 \% \mathrm{CI}=61 \%-92 \%)$ and negative predictive value was $92 \%(95 \% \mathrm{CI}=84 \%-98 \%)$. Two-hour lactate clearance also outperforms other markers commonly used in critical care such as baseline lactate $($ AUROC $=0.46), 2$-hour base excess $($ AUROC $=0.66)$, shock index $($ AUROC $=0.61)$ and MAP $(A U R O C=0.75)$. Two-hour lactate measurements produced AUROC of 0.84 but its sensitivity and specificity were inferior to LACT-2h-clearance given that a cut off of 2.5 $\mathrm{mg} / \mathrm{dL}$ returned a sensitivity of $82 \%$ but a specificity of only $64 \%$.
Variables identified by the backward logistic regression model as significantly correlated with negative outcome were LACT-2h-clearance less than 15\%, 2-hour lactate and MAP less than 90 at presentation. The log likelhood ratio of LACT-2h-clearance less than 15\%, was $40.08(P<.001)$. Other laboratory values, catacolamine use, age, sex, and comorbidites did not predict negative outcome in this model.

\section{Discussion}

The most important result of the present investigation was that LACT-2h-clearance can be feasible and clinically useful 
as a predictive tool in cardiorespiratory insufficiency. Under the experimental conditions of this study it seems that a cut-off of $<15 \%$ LACT-2h-clearance is predictive of negative outcome. This measure proved robust even when lactate levels were only mildly elevated at baseline $(<3 \mathrm{mmol} / \mathrm{L})$.

Lactate clearance deserves the same diagnostic relevance of other noninvasive markers of $\mathrm{O}_{2}$ delivery/consumption/demand mismatch. While tissue $\mathrm{pH}, \mathrm{O}_{2}$-saturation, $\mathrm{PCO}_{2}$, and (prospectively) NADH monitoring could offer a precise "local" picture of cellular dysoxia [16], lactate does not. Nevertheless the systematic checking of 2-hour lactate clearance could be used to tailor the therapy in many cases of cardiac or respiratory insufficiency. It seems to mirror quite precisely hepatic and renal function and perfusion $[2,17]$ in many critically ill patients. It cannot be substituted by any other single parameter derived from arterial blood gas analysis [18]. Reduced $\mathrm{O}_{2}$-delivery is compatible with aerobic respiration if oxygen consumption is simultaneously decreased; this is not an acidifying process "per se" [19]. On the other hand, there are definite "timewindows" in septic syndromes, where the hyper metabolism produces high blood lactate concentrations, in patients who are not yet acidotic. On the contrary, pure acute cardiac or respiratory insufficiencies can be considered acid and lactate producing syndromes, which are mostly associated with reduced acid and lactate removal. Moreover the effects of some therapies (fluid management, inotropes, ventilation, etc.) are not always predictable, due to intrinsic myocardial $\mathrm{O}_{2}$-consumption [20] and heart/lung/metabolism interactions. Often even invasive hemodynamic parameters can be puzzling.

Our patient cohort is made up predominantly of elderly patients with multiple comorbidities, which is representative of an increasingly large segment of the general population and of ICU patients. In such patients invasive ventilation is often better avoided if possible [21]. The decision to include patients with either cardiac and/or respiratory insufficiency was based on the frequent coexistence of these pathologies and the difficulty in precisely differentiating these entities in the emergency department. A robust early prognostic marker, such as LACT-2h-clearance, to identify patients with acute cardiorespiratory insufficiency at high risk of negative outcome irrespective of the ultimate diagnosis is an attractive clinical tool. In keeping with EGDT LACT$2 \mathrm{~h}$-clearance may provide a target for clinicians to tailor more aggressive therapy for patients at higher risk of negative outcome. Of equal importance is the identification of patients in whom the current therapy is working and who are likely to have a positive outcome. To our knowledge our work is the first to examine the prognostic value of lactate at 2 hours in this increasingly large patient population. Indeed the cut off of $15 \%$ clearance of serum lactate at 2 hours is lower and measured significantly sooner than the bulk of studies investigating the prognostic value of lactate. This is because much of the work in this field has been in relation to septic patients who respond more slowly to therapy than cardiorespiratory patients.

\section{Study Limitations}

This study has several limitations. First of all, there is a "lag phase" between the initial resolution of the cardiorespiratory crisis and the descending slope of lactate concentration. This is due to a "wash-out" phenomenon linked to flow restoration, plus the $\mathrm{O}_{2}$-debt which must be repaid by the liver and kidneys before they can restart the cellular "machinery." Second, it should be considered that the fast-responder organs are usually pouring their lactate into the blood well before it is detectable on any arterial sample, so that a precise evaluation should be done drawing the effluent blood of each organ (e.g., internal jugular or coronary veins). Third, the lactate/pyruvate $(=\mathrm{NADH} / \mathrm{NAD})$ ratio is not routine evaluation, but should be done whenever a hypermetabolic state is suspected [22, 23]. Fourth, there are some drugs and inherited metabolic syndromes which can complicate the picture. In particular inotropic agents are known to increase serum lactate levels. However, there was no correlation in our study between the use of catecholamines and negative outcome as evidenced by the logistic regression analysis.

We do not have data to assess how closely the care team complied with standard protocols and although the same team cared for all patients in the study, there may have been slight variability in the approach by individual clinicians who were in charge of the patients regarding parameters such as inotrope dosage or ventilator settings. These changes may have lead to differences in the quality of care provided, which could introduce a degree of bias into the results. Finally the study included patients with cardiorespiratory failure rather than the patients with a specific pathological diagnosis. Although the results indicate that LACT-2h-clearance predicts outcome irrespective of the underlying pathology the study was not adequately powered to perform subgroup analysis. A larger validation study would be necessary to confirm the predictive values suggested by the current data in specific patient subgroups with purely cardiac or respiratory pathology.

\section{Conclusions}

The systematic monitoring of lactate clearance at 2 hours should be clinically useful in cases of acute cardiac or respiratory insufficiency to identify patients at high risk of negative outcome and, potentially, to increase the intensity of the therapeutic approach. Finally, a 2-hour lactate clearance $>15 \%$ is highly predictive of positive outcome and may reassure clinicians that the therapeutic approach is appropriate. However, larger, prospective randomized studies will be needed to validate this hypothesis.
Abbreviations
LACT-2h-clearance: (Lactate start - lactate 2 hour)/ lactate start (\%)
LACT-6h-clearance: (Lactate start - lactate 6 hour)/ lactate start $(\%)$
APACHE II: $\quad$ Acute Physiology and Chronic Health Evaluation II 


\begin{tabular}{|c|c|}
\hline BP: & Blood Pressure \\
\hline BPM: & Beats Per Minute \\
\hline CI: & Confidence Interval. \\
\hline COPD: & $\begin{array}{l}\text { Chronic Obstructive Pulmonary } \\
\text { Disease }\end{array}$ \\
\hline ED: & Emergency Department \\
\hline EGDT: & Early Goal Directed Therapy \\
\hline $\mathrm{FiO}_{2}$ : & Inspired Fraction of Oxygen \\
\hline GCS: & Glasgow Coma Score \\
\hline $\mathrm{Hb}:$ & Hemoglobin \\
\hline HDU: & High Dependency Unit \\
\hline Hct: & Hematocrit \\
\hline ICU: & Intensive Care Unit \\
\hline MAP: & Mean Arterial Pressure \\
\hline NPPV: & $\begin{array}{l}\text { Noninvasive Positive Pressure } \\
\text { Ventilation }\end{array}$ \\
\hline $\mathrm{PaCO}_{2}$ : & $\begin{array}{l}\text { Partial Pressure of Arterial Carbon } \\
\text { Dioxide }\end{array}$ \\
\hline ROC curve: & $\begin{array}{l}\text { Receiver Operating Characteristic } \\
\text { curve }\end{array}$ \\
\hline $\mathrm{SpO}_{2}$ : & Oxygen Saturation \\
\hline WBC: & White Blood Cells \\
\hline
\end{tabular}

\section{References}

[1] B. A. Mizock and J. L. Falk, "Lactic acidosis in critical illness," Critical Care Medicine, vol. 20, no. 1, pp. 80-93, 1992.

[2] J. Levraut, C. Ichai, I. Petit, J.-P. Ciebiera, O. Perus, and D. Grimaud, "Low exogenous lactate clearance as an early predictor of mortality in normolactatemic critically ill septic patients," Critical Care Medicine, vol. 31, no. 3, pp. 705-710, 2003.

[3] J. Bakker, M. Coffernils, M. Leon, P. Gris, and J.-L. Vincent, "Blood lactate levels are superior to oxygen-derived variables in predicting outcome in human septic shock," Chest, vol. 99, no. 4, pp. 956-962, 1991.

[4] H. B. Nguyen, E. P. Rivers, B. P. Knoblich et al., "Early lactate clearance is associated with improved outcome in severe sepsis and septic shock," Critical Care Medicine, vol. 32, no. 8, pp. 1637-1642, 2004.

[5] G. Bernardin, G. Guiottoc, F. Paladinoc, and F. Schiraldic, "Blood pressure and arterial lactate level are early indicators of short-term survival in human septic shock," Intensive Care Medicine, vol. 22, no. 1, pp. 17-25, 1996.

[6] L. J. Kaplan and J. A. Kellum, "Initial pH, base deficit, lactate, anion gap, strong ion difference, and strong ion gap predict outcome from major vascular injury," Critical Care Medicine, vol. 32, no. 5, pp. 1120-1124, 2004.

[7] F. Valenza, G. Aletti, T. Fossali et al., "Lactate as a marker of energy failure in critically ill patients: hypothesis," Critical Care, vol. 9, no. 6, pp. 588-593, 2005.

[8] B. Levy, S. Gibot, P. Franck, A. Cravoisy, and P.-E. Bollaert, "Relation between muscle $\mathrm{Na}+\mathrm{K}+$ ATPase activity and raised lactate concentrations in septic shock: a prospective study," Lancet, vol. 365, no. 9462, pp. 871-875, 2005.

[9] S. Del Gaudio, S. Greco, G. Guiotto, F Paladino, F Schiraldi, and V. Antonaglia, "How early is enough in lactate monitoring?” Critical Care, vol. 10, supplement 1, p. 58, 2006.

[10] E. P. Rivers, H. B. Nguyen, S. Havstad et al., "Early goaldirected therapy in the treatment of severe sepsis and septic shock," New England Journal of Medicine, vol. 345, no. 19, pp. 1368-1377, 2001.
[11] W. A. Knaus, E. A. Draper, D. P. Wagner, and J. E. Zimmerman, "APACHE II: a severity of disease classification system," Critical Care Medicine, vol. 13, no. 10, pp. 818-829, 1985.

[12] G. Teasdale and B. Jennett, "Assessment of coma and impaired consciousness. A practical scale," Lancet, vol. 2, no. 7872, pp. 81-84, 1974.

[13] J. Levraut, C. Ichai, I. Petit, J.-P. Ciebiera, O. Perus, and D. Grimaud, "Low exogenous lactate clearance as an early predictor of mortality in normolactatemic critically ill septic patients," Critical Care Medicine, vol. 31, no. 3, pp. 705-710, 2003.

[14] S. Carley, S. Dosman, S. R. Jones, and M. Harrison, "Simple nomograms to calculate sample size in diagnostic studies," Emergency Medicine Journal, vol. 22, no. 3, pp. 180-181, 2005.

[15] A. Flahault, M. Cadilhac, and G. Thomas, "Sample size calculation should be performed for design accuracy in diagnostic test studies," Journal of Clinical Epidemiology, vol. 58, no. 8, pp. 859-862, 2005.

[16] J. C. Puyana and M. R. Pinsky, "Searching for non-invasive markers of tissue hypoxia," Critical Care, vol. 11, no. 1, article 116, 2007.

[17] O. Clemmesen, P. Ott, and F. S. Larsen, "Splanchnic metabolism in acute liver failure and sepsis," Current Opinion in Critical Care, vol. 10, no. 2, pp. 152-155, 2004.

[18] F. Schiraldi, "Time to abandon base excess as a reliable index in the ICU?" International Journal of Intensive Care spring, vol. 27, 1995.

[19] J. Handy, "The origin and interpretation of hyperlactataemia during low oxygen delivery states," Critical Care, vol. 11, no. 1, article 104, 2007.

[20] J.-L. Vincent and D. De Backer, "Oxygen transport-the oxygen delivery controversy," Intensive Care Medicine, vol. 30, no. 11, pp. 1990-1996, 2004.

[21] J. E. Sevransky and E. F. Haponik, "Respiratory failure in elderly patients," Clinics in Geriatric Medicine, vol. 19, no. 1, pp. 205-224, 2003.

[22] D. Brealey, M. Brand, I. Hargreaves et al., "Association between mitochondrial dysfunction and severity and outcome of septic shock," Lancet, vol. 360, no. 9328, pp. 219-223, 2002.

[23] B. Levy, L.-O. Sadoune, A.-M. Gelot, P.-E. Bollaert, P. Nabet, and A. Larcan, "Evolution of lactate/pyruvate and arterial ketone body ratios in the early course of catecholaminetreated septic shock," Critical Care Medicine, vol. 28, no. 1, pp. 114-119, 2000. 


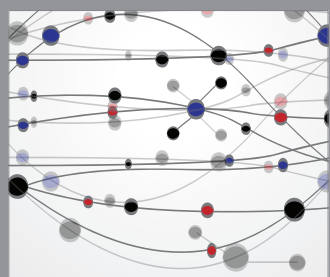

The Scientific World Journal
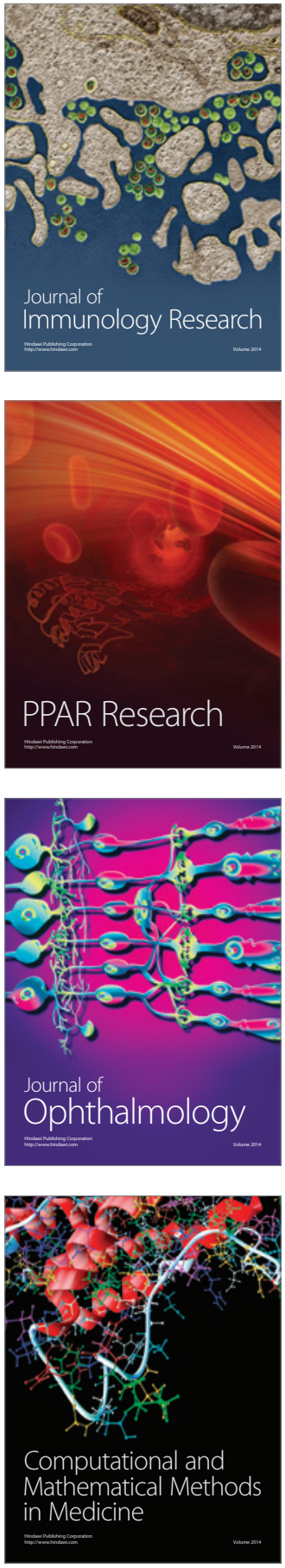

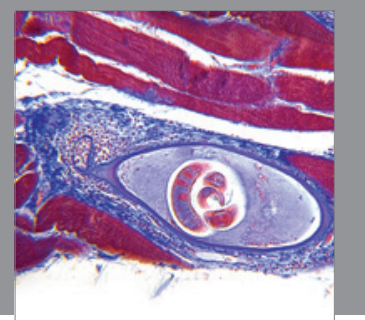

Gastroenterology

Research and Practice
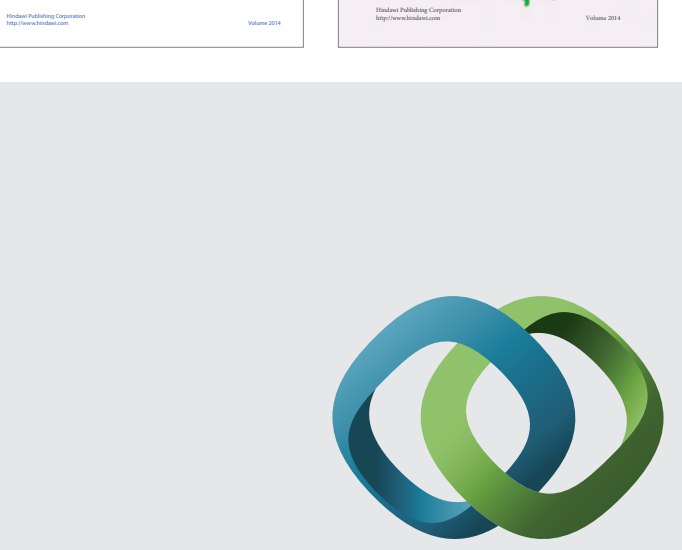

\section{Hindawi}

Submit your manuscripts at

http://www.hindawi.com
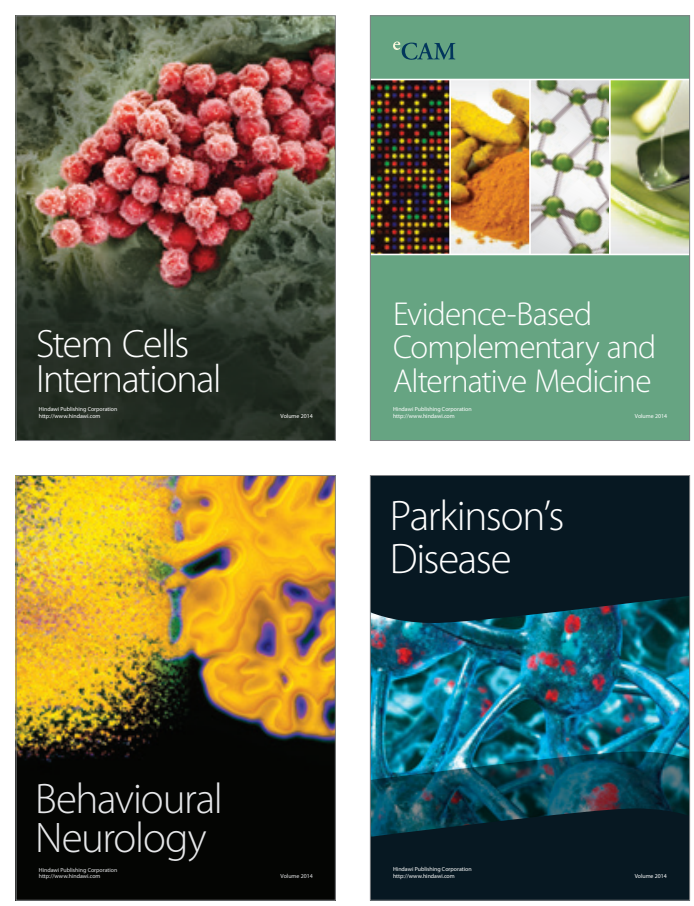

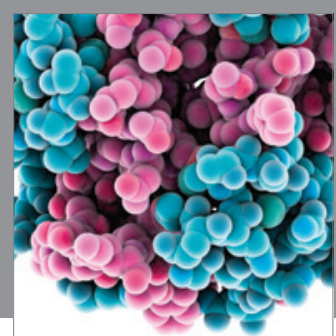

Journal of
Diabetes Research

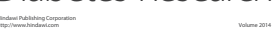

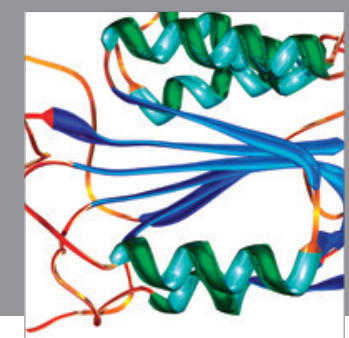

Disease Markers
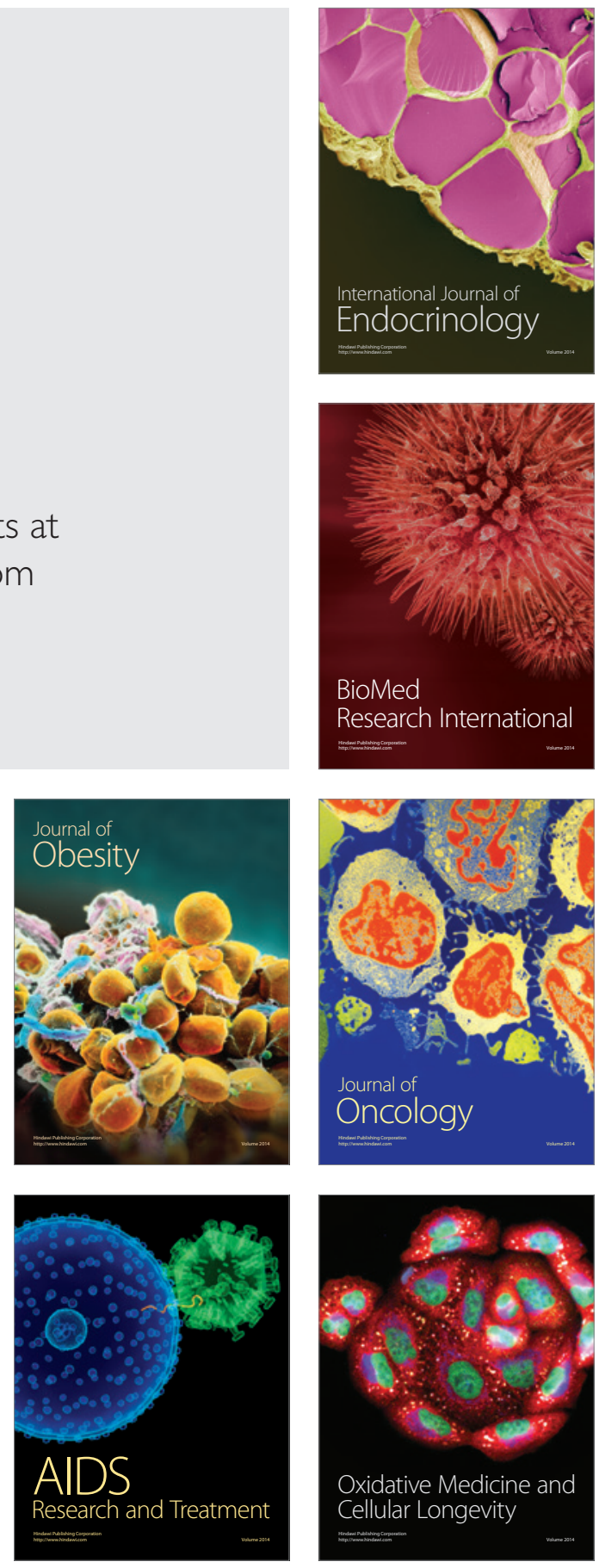